\title{
Comparaison de l'infectivité du coronavirus du syndrome respiratoire aigu sévère 2 chez les enfants et les adultes
}

\author{
Jared Bullard MD, Duane Funk MD, Kerry Dust PhD, Lauren Garnett BSc, Kaylie Tran BSc, Alex Bello PhD, \\ James E. Strong MD PhD, Santina J. Lee MD, Jillian Waruk PhD, Adam Hedley BSc, David Alexander PhD, \\ Paul Van Caeseele MD, Carla Loeppky PhD, Guillaume Poliquin MD PhD
}

— Citation : CMAJ 2021 April 26;193:E601-6. doi : 10.1503/cmaj.210263-f; diffusion hâtive le 9 avril 2021

Voir la version anglaise de l'article ici : www.cmaj.ca/lookup/doi/10.1503/cmaj.210263

\section{RÉSUMÉ}

CONTEXTE : Le rôle des enfants dans la propagation et la transmission communautaire du coronavirus du syndrome respiratoire aigu sévère 2 (SRAS-CoV-2) est encore mal compris. Nous visons à quantifier l'infectivité du SRAS-CoV-2 d'échantillons nasopharyngés provenant d'enfants comparativement à ceux provenant d'adultes.

MÉTHODES : Nous avons obtenu des écouvillons nasopharyngés de cas adultes et pédiatriques de la maladie à coronavirus 2019 (COVID-19) ainsi que de leurs contacts qui ont obtenu un résultat positif à la présence du SRASCoV-2 lors d'un test de dépistage au Manitoba entre les mois de mars et décembre 2020. Nous avons comparé la croissance virale en culture cellulaire, les valeurs de cycle seuil de test d'amplification en chaîne par polymérase couplé à une transcription inverse (RT-PCR) de l'enveloppe (E) du gène du SRAS-CoV-2 et de la dose infectieuse pour $50 \%$ de la culture tissulaire $\left(D I C T_{50} / \mathrm{mL}\right.$ ) entre les adultes et les enfants.

RÉSULTATS : Parmi les 305 échantillons positifs à la présence du SRAS-CoV-2 validés par RT-PCR, 97 échantillons provenaient d'enfants de 10 ans et moins, 78 échantillons d'enfants de 11-17 ans et 130 échantillons d'adultes ( $\geq 18$ ans). On a observé une croissance virale en culture dans $31 \%$ des échantillons, dont 18 (19\%) échantillons d'enfants de 10 ans et moins, 18 (23\%) d'enfants de 11-17 ans et 57 (44\%) d'adultes (enfants c. adultes, rapport de cotes 0,45 ; intervalle de confiance [IC] à $95 \% 0,28-0,72)$. Le cycle seuil était de 25,1 (IC à $95 \%$ 17,7-31,3) chez les enfants de 10 ans et moins, 22,2 (IC à $95 \% 18,3-29,0)$ chez les enfants de 11-17 ans et 18,7 (IC à $95 \% 17,9-30,4$ ) chez les adultes $(p<0,001)$. La DICT ${ }_{50} / \mathrm{mL}$ médiane était considérablement plus faible chez les enfants de 11-17 ans (316, écart interquartile [EI] 178-2125) que chez les adultes (5620, EI 1171$17800, p<0,001)$. Le cycle seuil était un indicateur exact d'une culture positive chez les enfants et les adultes (aire sous la courbe de la fonction d'efficacité du récepteur, 0,87, IC à $95 \% 0,81-0,93$ c. 0,89 , IC à $95 \% 0,83-$ $0,96, p=0,6)$.

INTERPRÉTATION : Comparés aux adultes, les enfants qui ont obtenu un résultat positif à un test de dépistage du SRAS-CoV-2 à l'aide d'un écouvillon nasopharyngé étaient moins susceptibles de présenter une croissance du virus en culture et obtenaient un cycle seuil plus élevé et une concentration virale moins élevée, indiquant que les enfants ne sont pas les principaux vecteurs de la transmission du SRAS-CoV-2. e coronavirus du syndrome respiratoire aigu sévère 2 (SRAS-CoV-2) et les mesures de santé publique non pharmacologiques (MNP) pour le contrôler ont eu des répercussions considérables sur la société. Les efforts de la santé publique déployés pour réduire la propagation de la maladie à coronavirus 2019 (COVID-19) ont employé plusieurs mesures de santé publique non pharmacologiques, dont la suspension de la présence physique en classe pour les enfants d'âge scolaire. On a pris ces décisions en grande partie sur la base d'observations historiques où les enfants jouaient un rôle important comme vecteurs de transmission dans les épidémies de virus respiratoires, comme la grippe ${ }^{1}$. Dans le cas du SRASCoV-2, le rôle des enfants dans la transmission demeure mal compris, étant donné le nombre restreint d'études qui 
présentent des données contradictoires ${ }^{2-9}$. La plupart des études étaient limitées à des enquêtes épidémiologiques à partir desquelles il est difficile de discerner la direction de la transmission ${ }^{3-7,9}$. Comme solution de rechange à ces éléments de preuve, certaines études ont plutôt investigué le rôle de la dynamique virale du SRAS-CoV-2, ici aussi avec des résultats hétérogènes. Parmi ces études, certaines ont montré des charges virales plus élevées dans le nasopharynx des cohortes pédiatriques sur la base des tests d'amplification en chaîne par polymérase alors que d'autres montraient des concentrations comparables de SRAS-CoV-2 chez les enfants et chez les adultes ${ }^{2,8,10,11}$. De plus, des données probantes associées à d'autres virus ont montré que de l'ARN viral peut demeurer détectable au-delà de l'infectivités,4. Un important indicateur de l'infectivité in vivo est la récupération de virus vivants sur une culture cellulaire. L'évaluation de cette dimension critique a fait défaut à la quasi-totalité des études sur le rôle des enfants dans la transmission du SRAS-CoV-2. Les données probantes démontrent que l'infectivité du SRAS-CoV-2 peut être prédite à l'aide des données disponibles, comme le cycle seuil du test d'amplification en chaîne par polymérase couplé à une transcription inverse (RT-PCR) ${ }^{12,13}$. Le cycle seuil est une mesure relative de la quantité de matériel génétique où des valeurs plus petites indiquent une présence accrue de matériel génétique dans l'échantillon.

Puisqu'un nombre croissant de provinces et de territoires examinent la question de la reprise ou de la poursuite des apprentissages à l'école, de l'ouverture des services de garde et de la tenue des activités parascolaires, une meilleure compréhension de la contribution relative des enfants et des adolescents à la transmission du SRAS-CoV-2, lorsqu'on la compare à celle des adultes, est essentielle. Cela est particulièrement important en raison de la probabilité croissante de la forme asymptomatique de l'infection dans ce groupe d'âge ${ }^{14,15}$. Notre objectif était de quantifier les taux de positivité des cultures de SRAS-CoV-2 issues d'écouvillons nasopharyngés positifs à la présence du virus démontrée par tests RT-PCR chez les enfants. Nous avons ensuite caractérisé la charge virale et les titres de spécimens dont la culture était positive et nous les avons comparés au groupe adulte.

\section{Méthodes}

\section{Conception de l'étude et population cible}

Dès le début du mois de juillet 2020, la province du Manitoba au Canada (population de 1,4 million de personnes) a vécu des éclosions de COVID-19 à large échelle. Dans un effort pour limiter la transmission, on a procédé à un dépistage complet des contacts des personnes infectées. Les définitions de cas de COVID-19 et de contacts des personnes infectées sont fournies à l'annexe 1 , accessible en anglais au www.cmaj.ca/lookup/doi/10.1503/ cmaj.210263/tab-related-content.

Nous avons obtenu des écouvillons nasopharyngés de patients atteints de la COVID-19 et de leurs contacts. Le Laboratoire provincial Cadham, le laboratoire de référence pour le dépistage du SRAS-CoV-2 au Manitoba, a procédé au dépistage des échantillons par test d'amplification en chaîne par polymérase couplé à une transcription inverse (RT-PCR). Les échantillons étaient prélevés aux centres de dépistage de la COVID-19 et étaient transportés au laboratoire dans un milieu de transport viral, généralement de 1-4 jours après le prélèvement. Nous entreposions les échantillons au laboratoire à $4{ }^{\circ} \mathrm{C}$ pour 24 heures jusqu'au moment de les tester, tel que décrit précédemment ${ }^{12}$. Nous testions tous les échantillons à l'aide d'une procédure de test développée au laboratoire afin de minimiser les variations de cycle seuil.

Du mois de mars au mois d'août 2020, nous avons soumis à une culture cellulaire tous les échantillons prélevés sur des enfants dont le test RT-PCR s'est avéré positif à la présence du SRAS-CoV-2. Comme le nombre de cas augmentait, on a fourni au Laboratoire de microbiologie national un échantillon de commodité de spécimens positifs pour l'analyse des cultures cellulaires. Nous avons sélectionné les échantillons de la semaine précédente pour nous assurer de leur fraîcheur afin de maximiser les rendements de la culture cellulaire. En novembre, nous avons sélectionné intentionnellement des spécimens pédiatriques présentant un cycle seuil de moins de 25 afin de confirmer nos observations préliminaires, soit que le taux de cultures positives de ces échantillons était plus faible que celui des échantillons adultes (tous les échantillons pédiatriques prélevés entre le 27 mars et le 8 novembre 2020). Nous avions déterminé précédemment que les valeurs de cycle seuil de moins de 25 offraient des rendements de culture plus élevés ${ }^{12}$. Simultanément, nous avons sélectionné un échantillon de commodité de spécimens adultes présentant une valeur de cycle seuil de 25 ou moins, à titre de comparaison. Avant l'analyse de culture cellulaire finale, nous avons sélectionné un échantillon de commodité de spécimens adultes, prélevés entre le 12 mars et le 14 décembre 2020 et provenant des mêmes régions sanitaires que les échantillons pédiatriques, pour la culture cellulaire.

\section{Paramètres}

Nos principaux paramètres étaient les taux de positivité des cultures, les valeurs de cycle seuil des tests RT-PCR, la dose infectieuse pour $50 \%$ de la culture tissulaire $\left(D I_{C} T_{50} / \mathrm{mL}\right)$, la charge virale (le log des copies d'ARN par $\mathrm{mL}$ ) et la période entre l'apparition des symptômes et le test de dépistage. Pour tous les échantillons positifs, nous avons obtenu les valeurs de cycle seuil du test RT-PCR du gène E de l'enveloppe du SRAS-CoV-2. Nous avons aussi obtenu les valeurs de cycle seuil du gène humain codant pour la ribonucléase (RNAse) $P$, un contrôle d'amplification interne endogène utilisé comme marqueur de la qualité de l'échantillon nasopharyngé.

L'essai DICT $_{50} / \mathrm{mL}$ est une méthode de quantification des titres de virus infectieux. Plus spécifiquement, il quantifie le volume de virus nécessaire pour tuer $50 \%$ des cellules d'une culture tissulaire, produisant ainsi un effet cytopathologique. On a entreposé la plupart des échantillons à $-80{ }^{\circ} \mathrm{C}$ pour 2 semaines avant de procéder à la culture. Le Laboratoire national de microbiologie a déterminé les titres viraux des 
échantillons (confinement biologique de niveau 4) à l'aide d'essais DICT $_{50} / \mathrm{mL}$ (la méthodologie détaillée est présentée à l'annexe 1). En bref, on a placé des échantillons dilués en séries sur des cellules Vero et incubés de $96-120$ heures à $37^{\circ} \mathrm{C}$ dans une atmosphère à $5 \%$ de $\mathrm{CO}_{2}$ avant de mesurer la $\mathrm{DICT}_{50}$.

La charge virale est habituellement mesurée comme le logarithme du nombre de copies du génome de l'ARN par millilitre ( $\log$ des copies d'ARN $/ \mathrm{mL}$ ), une valeur quantitative plus normalisée que les cycles seuil. Dans le cadre de cette étude, et pour quantifier la quantité d'ARN viral présent dans chaque échantillon, nous avons généré une courbe de concordance en utilisant une quantité connue d'ARN viral ou d'ADN copié qui a été dilué en série et utilisé en même temps comme échantillon de test afin de fournir une relation entre le cycle seuil et les copies de génome/mL (annexe 1).

Nous avons déterminé la date de l'apparition des symptômes à partir des dossiers de la santé publique, de l'épidémiologie, de la surveillance et du laboratoire. Nous avons aussi calculé le nombre de jours constituant la période entre l'apparition des symptômes et le moment du prélèvement de l'échantillon pour le dépistage, selon les dossiers du laboratoire (voir l'annexe 1).

\section{Analyse statistique}

Dans nos travaux précédents ${ }^{12}$, nous avons observé que les adultes présentaient un taux de cultures positives de $28,9 \%$. Par conséquent, nous avons besoin de 164 échantillons pédiatriques pour détecter une différence cliniquement significative (un taux de cultures positives plus bas de $33 \%$ à une puissance de 0,8 et $\alpha$ de 0,05 ) chez les enfants.

Nous présentons des données distribuées normalement accompagnées de moyennes et d'écarts-types et des données non normalement distribuées accompagnées de médianes et d'écarts interquartiles (EI). Nous avons évalué la normalité en employant le test de Kolmogorov-Smirnov. Nous avons procédé à des comparaisons entre les groupes à l'aide du test $t$ de Student ou du test Mann-Whitney et nous avons utilisé la méthode exacte de Fisher pour les données catégoriques. Nous avons comparé les médianes de groupes non paramétriques à l'aide de l'analyse de variance Kruskal-Wallis. Nous avons employé la régression logistique multivariée à l'aide d'erreurs types robustes afin de tester les indicateurs de cultures positives. Nous avons considéré les intervalles bilatéraux de valeur $p$ inférieurs à 0,05 comme étant significatifs. Nous avons réalisé l'analyse statistique à l'aide des logiciels Stata version 16.1 et GraphPad Prism 9.

\section{Approbation éthique}

L'étude a été réalisée conformément au protocole HS23906 (H2020:211) et approuvée par le comité d'éthique de la recherche de l'Université du Manitoba. Le comité d'éthique n'a pas jugé nécessaire d'obtenir le consentement éclairé puisque les échantillons ont été obtenus dans le cadre d'une prise en charge clinique de routine de la santé publique et n'ont pas été recueillis spécifiquement pour être intégrés dans la présente étude.

\section{Résultats}

Au cours de la période de l'étude, environ 360000 tests de dépistage d'écouvillons nasopharyngés ont été réalisés au Manitoba; de ceux-ci, 20000 étaient positifs à la présence du SRAS-CoV-2. Notre échantillon final comprenait 305 spécimens de culture, représentant $1,5 \%$ des échantillons positifs du Manitoba et 7,2\% (175 de 2440) des échantillons positifs provenant d'enfants. Des 175 échantillons de culture pédiatriques, 97 échantillons provenaient d'enfants de 10 ans et moins et 78 provenaient d'enfants de 11-17 ans; on a comparé ces échantillons à ceux de 130 spécimens adultes. Les caractéristiques démographiques, les cycles seuil et les charges d'ARN viral sont présentés au tableau 1 et au tableau 2. Nous avons réussi la culture du virus dans 93 des 305 échantillons (31\%), dont 57 des 130 échantillons adultes (44\%, IC à $95 \% 35 \%-53 \%$ ). En comparaison, nous avons procédé à une culture du virus dans seulement 18 des 97 échantillons chez les enfants de 10 ans et moins ( $19 \%$, IC à $95 \% 11 \%-28 \%, p<0,001)$ et 18 des 78 échantillons chez les enfants de $11-17$ ans (23\%, IC à $95 \% 14 \%-34 \%, p=0,003$ ). Le taux de cultures positives ne différait pas entre les enfants plus jeunes et ceux plus âgés $(p=0,5)$. Comparativement aux adultes, les enfants étaient $55 \%$ moins susceptibles de faire croître le virus vivant (rapport de cotes 0,45 , IC à $95 \% 0,28$ 0,72 ). Bien que la présence d'infections asymptomatiques était plus probable chez les enfants de 10 ans et moins $(47 / 97,48 \%)$ que chez les enfants de $11-17$ ans $(19 / 78,24 \%)$ ou que chez les adultes $(9 / 130,7 \%)(p<0,001$ pour toutes les comparaisons), tous les enfants de 17 ans et moins étaient également susceptibles de présenter une telle infection, peu importe que leur échantillon de culture soit positif ou négatif ( $42 \%$ c. $37 \%, p=0,9)$.

La qualité des échantillons nasopharyngés, comme démontré par les valeurs de cycle seuil du gène humain codant pour la ribonucléase (RNAse) $P$, était équivalente pour les 3 groupes d'âge $(p=0,6)$. Le cycle seuil du gène $E$ du SRAS-CoV-2 était plus bas chez les adultes $(18,7$. El 17,9-30,4) que chez les enfants de 10 ans et moins $(25,1$, El 17,7-31,3, $p<0,001)$ ou que chez les enfants de $11-17$ ans $(22,2$, El 18,3-29,0, $p=0,02)$ (tableau 1 et figure 1 ).

La dose infectieuse pour $50 \%$ de la culture tissulaire $\left(D_{I C T} / \mathrm{mL}\right.$ ) médiane était considérablement plus faible chez les enfants de 11-17 ans (316, El 178-2125) que chez les adultes $(5620$, El 1171-17800, $p<0,001)$, mais la différence entre les adultes et les enfants de 10 ans et moins (1171, El 316-5620, $p=0,1)$ n'a pas atteint le seuil de portée statistique (tableau 1 et figure 2).

Les échantillons pédiatriques de culture positive et négative étaient similaires, sauf pour leurs valeurs de cycle seuil et de log des copies d'ARN/mL (tableau 2). La médiane du cycle seuil était plus faible dans les échantillons de culture positive $(16,8$, El 16,3-18,8 que ceux de culture négative $(25,8$, El 20,7$31,9, p<0,001)$. La médiane du log des copies d'ARN/mL était plus élevée dans les échantillons de culture positive $(8,1$, EI 7,4-8,2) que ceux de culture négative (5,2, El 3,2-6,8, 
Tableau 1 : Mesures de l'infectivité du SRAS-CoV-2 chez les enfants et les adultes

\begin{tabular}{|c|c|c|c|c|}
\hline Variables & $\begin{array}{l}\text { Enfants de } \leq 10 \text { ans } \\
\qquad n=97\end{array}$ & $\begin{array}{l}\text { Enfants de } 11-17 \text { ans } \\
\qquad n=78\end{array}$ & $\begin{array}{l}\text { Adultes } \\
n=130\end{array}$ & Valeur $p$ \\
\hline Asymptomatique, $\mathrm{n}^{\text {bre }}(\%)$ & $47(48)$ & $19(24)$ & $9(7)$ & $<0,001 \S$ \\
\hline Culture positive, $\mathrm{n}^{\text {bre }}$ (\%, IC à $\left.95 \%\right)$ & $18(19,11-28)$ & $18(23,14-34)$ & $57(44,35-53)$ & $<0,0019$ \\
\hline $\begin{array}{l}\text { Période entre l'apparition des symptômes } \\
\text { et le test de dépistage, médian (EI), jours }\end{array}$ & $1(1-4)$ & $2(1-3,5)$ & $2(1-4)$ & 0,6 \\
\hline Cycle seuil ${ }^{\star}$, médian (EI) & $25,1(17,7-31,3)$ & $22,2(18,3-29,0)$ & $18,7(17,9-30,4)$ & $<0,001^{\star \star}$ \\
\hline RNase P†, moyenne \pm E.-T. & $25,7 \pm 2,8$ & $26,1 \pm 2,6$ & $26,1 \pm 2,0$ & 0,6 \\
\hline $\mathrm{DICT}_{50} / \mathrm{mL} \ddagger$, médian (EI) & $1171(316-5620)$ & $316(178-2125)$ & $5620(1171-17800)$ & $<0,001 \dagger \dagger$ \\
\hline $\begin{array}{l}\text { Log du nombre de copies de l'ARN/mL, } \\
\text { médian (EI) }\end{array}$ & $5,4(3,5-7,8)$ & $6,4(4,2-7,6)$ & $7,5(5,2-8,3)$ & $<0,001 \ddagger \ddagger$ \\
\hline \multicolumn{5}{|c|}{ 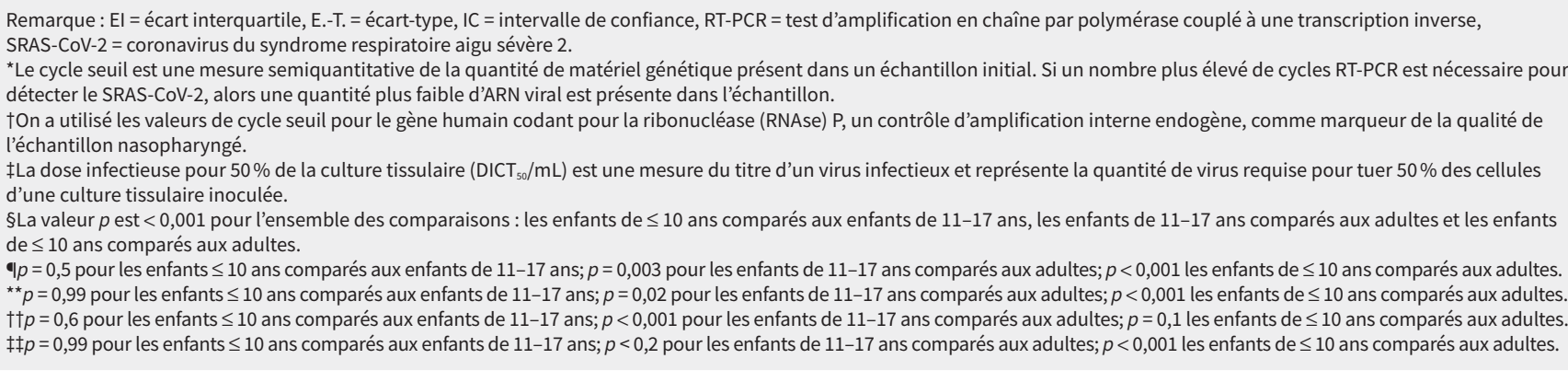 } \\
\hline
\end{tabular}

Tableau 2 : Mesure de l'infectivité du SRAS-CoV-2A dans des échantillons pédiatriques positifs à la culture et négatifs à la culture

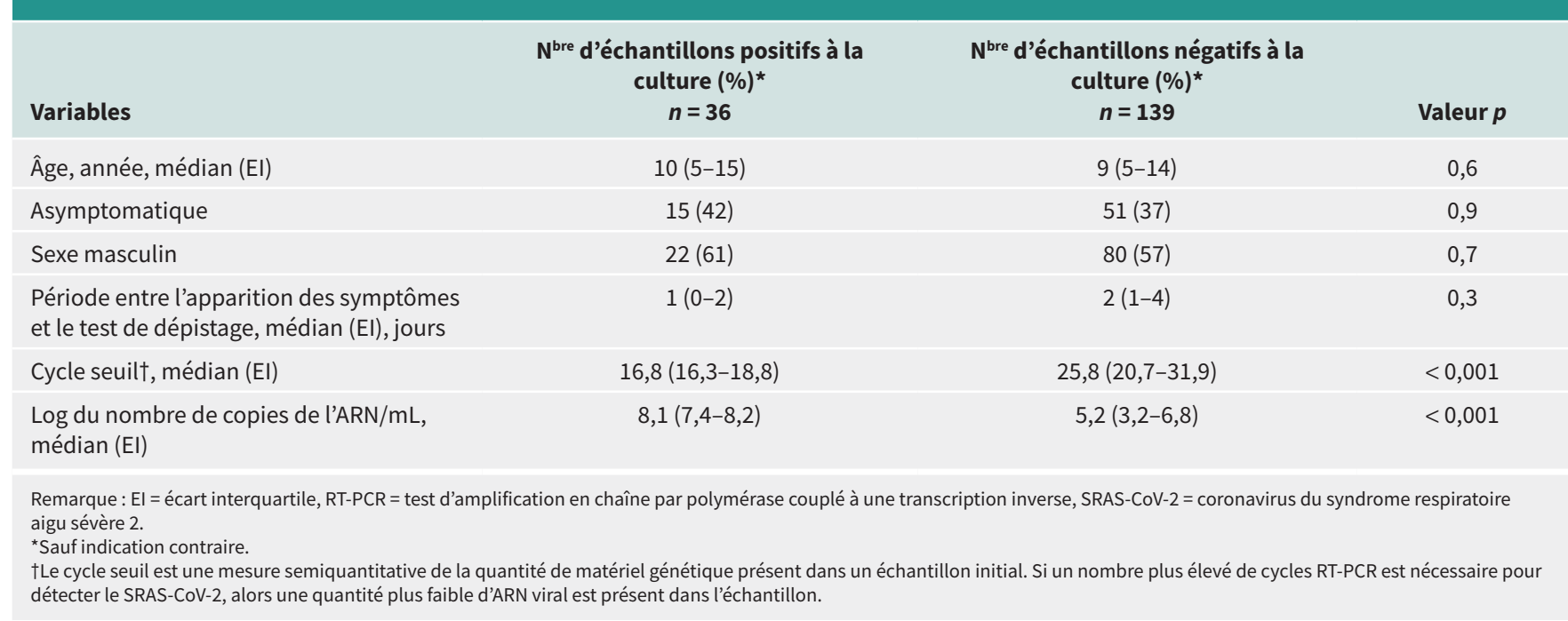

$p<0,001)$. Cependant, la médiane de la période entre l'apparition des symptômes et le moment du prélèvement de l'échantillon pour le dépistage ne différait pas entre le groupe de culture positive (1 jour, El 0-2 jours) et celui de culture négative (2 jours, EI 1-4 jours, $p=0,3$ ). La probabilité d'obtenir une culture positive variait selon la période entre l'apparition des symptômes et le moment du prélèvement de l'échantillon pour le dépistage, la probabilité d'une culture positive étant la plus élevée parmi les spécimens prélevés entre 4 et 6 jours après l'apparition des symptômes, alors que le cycle seuil montrait moins de variations entre les mêmes périodes (figure 3).

L'analyse de la fonction d'efficacité du récepteur (ROC) du cycle seuil pour discriminer entre les enfants présentant ou non une culture virale positive a montré une aire sous la courbe (ASC) de la fonction d'efficacité du récepteur de 0,87 (IC à 


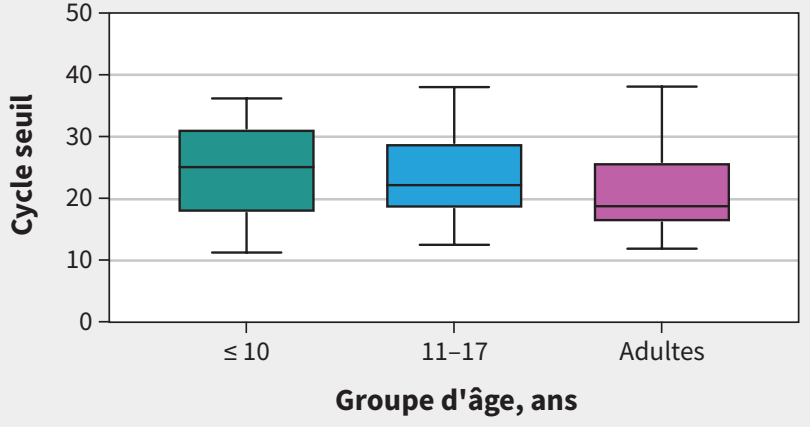

Figure 1 : Valeurs de cycle seuil du test d'amplification en chaîne par polymérase couplé à une transcription inverse de l'enveloppe du gène du coronavirus du syndrome respiratoire aigu sévère 2 selon le groupe d'âge. Les échantillons des adultes présentaient une valeur de cycle seuil considérablement plus faible (18,7, écart interquartile [EI] 17,9-30,4) que celle des enfants de $\leq 10$ ans $(25,1$, El $17,7-31,3, p<0,001)$ et ceux de $11-17$ ans (22,2, El 18,3-29,0, $p=0,02)$.

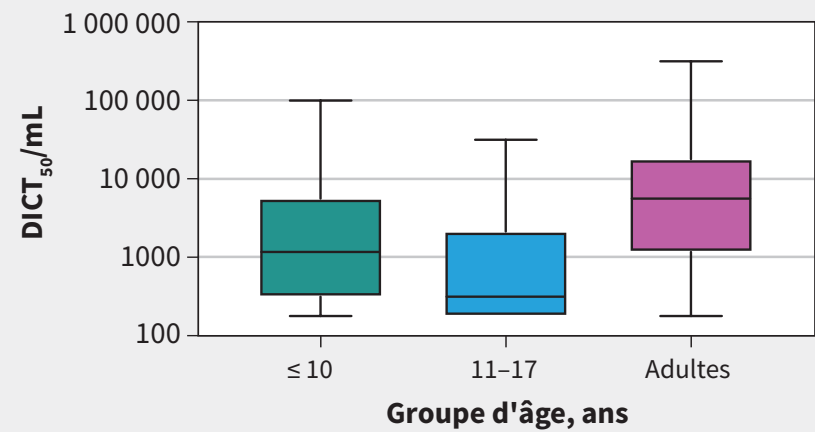

Figure 2 : Dose infectieuse pour $50 \%$ de la culture tissulaire $\left(\mathrm{DICT}_{50} / \mathrm{mL}\right)$ selon le groupe d'âge. Les échantillons des adultes présentaient une valeur de $\mathrm{DICT}_{50} / \mathrm{mL}$ considérablement plus élevée (5620, écart interquartile [EI] 1171-17 800) que les enfants de 11-17 ans (316, El 178-2125, $p<0,001$ ), mais elle n'était pas considérablement plus élevée que celle des enfants de $\leq 10$ ans (1171, El 316-5620, $p=0,1$ ).

$95 \%$ 0,81-0,93) (annexe 1, figure supplémentaire 1). La spécificité d'un cycle seuil de 23 était de $97,2 \%$ (IC à $95 \%$ 85,8\%-99,9\%) (annexe 1, tableau supplémentaire 1). Des résultats similaires ont été observés chez les adultes (ASC 0,89 , IC à $95 \% 0,83-0,96, p=0,6 \mathrm{c}$. les enfants) (annexe 1, figure supplémentaire 1 , tableau supplémentaire 2). La période entre l'apparition des symptômes et le moment du prélèvement de l'échantillon pour le dépistage n'était pas aussi exacte que le cycle seuil pour discriminer entre les échantillons présentant ou non une culture virale positive (enfants ASC 0,67, IC à $95 \%$ 0,55-0,79 c. adultes, ASC 0,78, IC à $95 \% 0,68-0,88, p=0,2$ ) (annexe 1, figure supplémentaire 2), avec une spécificité de $100 \%$ (IC à $95 \% 84,5 \%-100 \%$ ) pour une période entre l'apparition des symptômes et le moment du prélèvement de l'échantillon pour le dépistage de plus de 6 jours. Il est à noter que notre échantillonnage comptait seulement 8 patients dont la période entre l'apparition des symptômes et le moment du prélèvement de l'échantillon pour le dépistage était de 6 jours

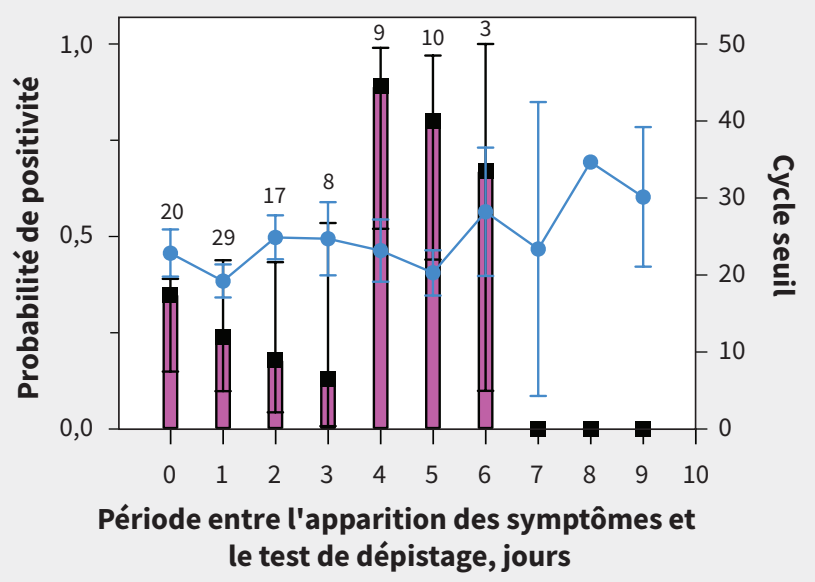

Figure 3 : Période entre l'apparition des symptômes et le test de dépistage (en jours), la valeur moyenne du cycle seuil du test d'amplification en chaîne par polymérase couplé à une transcription inverse de l'enveloppe du gène du coronavirus du syndrome respiratoire aigu sévère 2 et la probabilité d'une culture virale positive dans des échantillons pédiatriques. La probabilité d'une culture du SRAS-CoV-2 est illustrée par les barres roses. Les lignes noires représentent les intervalles de confiance à $95 \%$. Les valeurs de cycle seuil sont montrées par la ligne bleue, les cercles représentant les médianes et les barres bleues représentant les intervalles de confiance à $95 \%$. Les nombres inscrits audessus de la barre rose indiquent le nombre d'échantillons par jour.

ou plus, remettant en question la précision des résultats pour déterminer une limite à cette période en lien avec la positivité des cultures cellulaires et la possible infectivité en raison d'un manque de données.

Pour les échantillons pédiatriques, la régression logistique multivariée a montré que le cycle seuil était indépendant de l'indicateur de la culture positive (rapport de cotes 0,81 , IC à $95 \% 0,69-0,94)$, mais la période entre l'apparition des symptômes et le moment du prélèvement de l'échantillon pour le dépistage, l'âge et le sexe ne l'étaient pas (tableau 3).

Dans une analyse additionnelle, nous n'avons observé aucune différence dans les taux de culture positive entre les enfants de 0-4 ans et ceux de 5-10 ans. Le niveau de virus (selon la $\mathrm{DICT}_{50} / \mathrm{mL}$ ) ne variait pas au sein des échantillons de culture positive parmi les enfants de ces 2 groupes d'âge.

\section{Interprétation}

Nos résultats montrent que les échantillons de 175 enfants de 17 ans et moins présentaient une probabilité à moitié moindre de contenir des particules virales pouvant être cultivées que les échantillons provenant d'adultes. Lorsque la culture du SRAS-CoV-2 était réussie, la dose infectieuse pour $50 \%$ de la culture tissulaire $\left(\mathrm{DICT}_{50} / \mathrm{mL}\right)$ médiane était considérablement plus faible pour les échantillons pédiatriques que pour les adultes, indiquant qu'une moins grande quantité de particules virales viables étaient présentes. De plus, le taux de culture positive des échantillons d'enfants de 10 ans et moins était considérablement plus faible que celui des enfants de 
Tableau 3 : Modèle de régression logistique multivariée de mesures associées à une culture virale positive d'échantillons pédiatriques

\section{Variables}

Cycle seuil ${ }^{*}$

Période entre l'apparition

des symptômes et le test

de dépistage

Âge

Sexe

RNase P†
Rapport de cotes ajusté (IC à $95 \%$ )

$0,81(0,69-0,94)$

$0,90(0,64-1,27)$

$1,13(0,97-1,31)$

$2,18(0,48-9,87)$

$0,69(0,48-1,00)$
Remarque : IC = intervalle de confiance, RT-PCR = test d'amplification en chaîne par polymérase couplé à une transcription inverse, SRAS-CoV-2 = coronavirus du syndrome respiratoire aigu sévère 2 .

*Le cycle seuil est une mesure semiquantitative de la quantité de matériel génétique présent dans un échantillon initial. Si un nombre plus élevé de cycles RT-PCR est nécessaire pour détecter le SRAS-CoV-2, alors une quantité plus faible d'ARN viral est présente dans l'échantillon.

tOn a utilisé les valeurs de cycle seuil pour le gène humain codant pour la ribonucléase (RNAse) P, un contrôle d'amplification interne endogène, comme marqueur de la qualité de l'échantillon nasopharyngé.

11-17 ans ou que celui des adultes. Ces résultats illustrent que la positivité des tests d'amplification en chaîne par polymérase couplé à une transcription inverse (RT-PCR) ne correspond pas nécessairement à une culture positive puisque la positivité du test RT-PCR à elle seule ne permet pas de faire la distinction entre le virus vivant chez un patient infectieux et des particules d'ARN virale résiduelles chez un patient qui n'est peut-être plus infectieux.

Nous avons constaté que la valeur de cycle seuil était hautement prédictive de la positivité des cultures. En revanche, la période entre l'apparition des symptômes et le test de dépistage ne permettait pas de discriminer entre les enfants présentant une culture positive de ceux présentant une culture négative. Ainsi, chez les enfants qui ont obtenu un test de dépistage positif au SRAS-CoV-2 par RT-PCR, la valeur du cycle seuil pourrait avoir une incidence sur la durée de l'isolement, sachant que cette valeur peut être utile pour déterminer l'infectivité potentielle d'un enfant.

Ces résultats sont à l'opposé des observations associées à d'autres virus respiratoires pour lesquels une infection et une transmission efficaces chez les enfants annoncent souvent une transmission communautaire à grande échelle. Cependant, ces résultats sont cohérents avec les études épidémiologiques qui montrent une transmission limitée du SRASCoV-2 chez les enfants de moins de 10 ans $^{16,17}$. Une récente étude de séroprévalence réalisée en Allemagne a démontré que les enfants, surtout ceux de 1-10 ans, présentent une séropositivité considérablement plus faible que leurs parents, rendant moins probables les infections asymptomatiques non détectées chez les enfants ${ }^{18}$. Une méta-analyse a révélé une susceptibilité moins élevée au SRAS-CoV-2 chez les enfants qui ne seraient donc pas des vecteurs de transmission communautaire de même ampleur que les adultes ${ }^{19}$. Le coronavirus du syndrome respiratoire aigu sévère 2 présente aussi un taux de reproduction (RO) excessivement dispersé, indiquant que sa dynamique de transmission est fondamentallement différente de celle des épidémies saisonnières de virus respiratoires ${ }^{20-22}$. La surdispersion fait référence à la grande variation de la distribution du nombre de transmissions secondaires au niveau individuel qui peut mener aux incidents dits " superpropagateurs » ${ }^{21}$.

D'autres ont étudié la capacité de faire croître des virus vivants à partir des échantillons pédiatriques, la référence standard pour le diagnostic microbiologique. L'Huillier et ses collègues ont fait croître des virus à partir d'une plus grande proportion d'échantillons pédiatriques que celle de notre étude ( $52 \% ; 12 / 23$, c. $31 \%$ pour tous nos échantillons combinés) ${ }^{11}$. Leurs résultats en lien avec la période entre l'apparition des symptômes et le test de dépistage étaient semblables à nos observations et l'IC à $95 \%$ de nos résultats se superposait presque qu'au leur, indiquant que la taille plus petite de leur échantillon pourrait être responsable de la plus grande proportion de cultures positives observée dans leurs résultats. Cependant, un examen plus approfondi des données de L'Huillier a révélé que, en accord avec notre présente étude, la positivité des cultures variait selon l'âge, de telle sorte que la culture des virus a pu être réalisée sur 4 des $11(36,4 \%)$ enfants de 10 ans et moins, mais 8 des $12(66,6 \%)$ enfants de 11 ans et plus. Reconnaissant que le cycle seuil est un substitut limité à la charge virale, d'autres études ont essayé de quantifier davantage cette charge virale chez les enfants en employant le log des copies d'ARN/mL basé sur des courbes normalisées. Bien que cette approche améliore la capacité d'effectuer des comparaisons interlaboratoires et temporelles des données, elle n'en demeure pas moins une mesure de substitut à la charge virale viable, elle ne peut prédire la possibilité de récupérer des virus vivants et elle peut être induite en erreur par l'excrétion de matériel génétique viral non infectieux. De ce fait, les conclusions tirées des mesures de charge virale dérivées des données de cycle seuil sont sujettes à des limitations substantielles. Nous avons quantifié la présence de virus à l'aide de la dose infectieuse pour $50 \%$ de la culture tissulaire $\left(\mathrm{DICT}_{50} / \mathrm{mL}\right)$, laquelle fournit un pouvoir de discrimination additionnel comparativement aux méthodes qui limitent l'analyse à la simple présence ou absence d'effet cytopathologique ${ }^{11}$.

L'observation d'une valeur de cycle seuil supérieure à 23, indiquant une probabilité considérablement moins importante de récupérer les virus vivants, vaut la peine d'être examinée dans une étude de plus grande envergure. Cette valeur est en accord avec nos travaux précédents qui ont démontré une capacité atténuée de faire croître des virus vivants dans des échantillons prélevés chez des adultes où la valeur de cycle seuil était supérieure à $24^{12}$. Finalement, on devrait entreprendre de définir une période limite robuste fondée sur les symptômes comme indicateur d'une culture cellulaire positive, bien que le dépistage se déroule souvent très tôt après l'apparition des symptômes. Il pourrait s'avérer difficile de répondre à cette question dans l'environnement actuel du dépistage de la COVID-19. 


\section{Limites de l'étude}

Nos résultats peuvent s'expliquer autrement. Des variations génériques virales pourraient jouer un rôle; cependant, la surveillance génomique montre que les échantillons représentaient les différentes lignées mondiales présentes lors de la vague initiale et des vagues subséquentes au Manitoba. Le prélèvement d'échantillons peut être difficile chez les enfants, entraînant des spécimens sous-optimaux. L'absence de différences significatives dans les valeurs de cycle seuil de la RNAse $P$ (un contrôle d'amplification interne endogène) suggère cependant que la qualité des échantillons était comparable pour tous les groupes d'âge. On a aussi considéré la dégradation des échantillons lors de l'entreposage, influençant la probabilité de récupération de particules virales, mais la période entre le prélèvement et la culture cellulaire était comparable pour tous les groupes d'âge, rendant cette possibilité peu probable.

Bien que les jeunes enfants présentaient des périodes similaires entre l'apparition des symptômes et le test de dépistage, ils peuvent atteindre le moment d'infectivité maximale à des temps postexposition différents des adolescents et des adultes. Notre épidémiologie locale (données non publiées, 2021) ne soutient pas cet argument puisque les cas pédiatriques de la COVID-19 sont cohérents avec la transmission communautaire ${ }^{23,24}$. Il est possible que les enfants étaient à un point différent des adolescents et des adultes sur leur trajectoire virale au moment du prélèvement. Comme on n'a prélevé qu'un seul échantillon, il n'est pas possible de déterminer la tendance longitudinale de la valeur de cycle seuil en fonction du temps écoulé depuis le prélèvement. Une analyse de régression (données non présentées ici) des échantillons adultes et enfants n'a montré aucune corrélation entre la période entre l'apparition des symptômes et le test de dépistage et la valeur de cycle seuil ou de DICT ${ }_{50} / \mathrm{mL}$. Il ne faut pas oublier qu'un biais sur l'apparition des symptômes est possible et que ceux-ci peuvent être discrets chez les enfants, aggravant ainsi ce biais, mais cette probabilité est également distribuée parmi tous les patients. Finalement, nous ne pouvons être certains que nos résultats s'appliquent aux nouveaux variants du SRAS-CoV-2 qui ont démontré un plus haut degré d'infectivité, car ces variants n'étaient pas en circulation au cours de la période visée par l'étude.

\section{Conclusion}

Nous avons démontré que le SRAS-CoV-2 prélevé sur des échantillons pédiatriques pouvait être mis en culture moins fréquemment que celui prélevé sur des échantillons adultes et lorsque la culture était réussie, la quantité de virus viable était considérablement moindre. Ces données, tout comme notre épidémiologie locale, indiquent que les enfants ne semblent pas être les vecteurs principaux de la transmission du SRAS$\mathrm{CoV}-2$. Nos résultats ont d'importantes implications cliniques et de santé publique. Si les jeunes enfants sont moins en mesure de transmettre le virus infectieux, la fréquentation des services de garde et des écoles ainsi que la pratique prudente d'activités parascolaires peuvent se poursuivre de façon sécuritaire si les mesures de précaution appropriées sont mises en place et avec un risque moindre qu'initialement anticipé pour les éducateurs, les enseignants et le personnel de soutien. Étant donné les difficultés de garder les enfants en isolement dans l'environnement familial et l'effet significatif d'un isolement prolongé sur le développement de l'enfant et le rôle parental (comme la perte d'emploi ou de revenu), un outil robuste visant à diminuer la durée ou la nécessité de la quarantaine pourrait être une importante avancée en santé publique.

\section{Références}

1. Reichert TA, Sugaya N, Fedson DS, et al. The Japanese experience with vaccinating schoolchildren against influenza. N Engl J Med 2001;344:889-96.

2. Baggio S, L'Huillier AG, Yerly S, et al. SARS-CoV-2 viral load in the upper respiratory tract of children and adults with early acute COVID-19. Clin Infect Dis 2020 Aug. 6; ciaa1157. doi: 10.1093/cid/ciaa1157. [Cyberpublication avant impression].

3. Guilamo-Ramos V, Benzekri A, Thimm-Kaiser M, et al. Reconsidering assumptions of adolescent and young adult SARS-CoV-2 transmission dynamics. Clin Infect Dis 2020; Sept. 7; ciaa1348. doi: 10.1093/cid/ciaa1348. [Cyberpublication avant impression].

4. Lopez AS, Hill M, Antezano J, et al. Transmission dynamics of COVID-19 outbreaks associated with child care facilities - Salt Lake City, Utah, AprilJuly 2020. MMWR Morb Mortal Wkly Rep 2020;69:1319-23.

5. Schmidt E, Steinhagen K, Rupp J. Heavy exposure of children aged 9-12 years with severe acute respiratory syndrome coronavirus 2 did not lead to infection. J Pediatric Infect Dis Soc 2020;9:620-1.

6. Stein-Zamir C, Abramson N, Shoob H, et al. A large COVID-19 outbreak in a high school 10 days after schools' reopening, Israel, May 2020. Euro Surveill 2020;25:2001352.

7. Szablewski CM, Chang KT, Brown MM, et al. SARS-CoV-2 transmission and infection among attendees of an overnight camp - Georgia, June 2020. MMWR Morb Mortal Wkly Rep 2020;69:1023-5.

8. Yonker LM, Neilan AM, Bartsch Y, et al. Pediatric severe acute respiratory syndrome coronavirus 2 (SARS-CoV-2): clinical presentation, infectivity, and immune responses. J Pediatr 2020;227:45-52 e5.

9. Zhang J, Litvinova M, Liang Y, et al. Changes in contact patterns shape the dynamics of the COVID-19 outbreak in China. Science 2020;368:1481-6.

10. Hurst JH, Heston SM, Chambers HN, et al. SARS-CoV-2 infections among children in the Biospecimens from Respiratory Virus-Exposed Kids (BRAVE Kids) study. Clin Infect Dis 2020; Nov. 3;ciaa1693. doi: 10.1093/cid/ciaa1693. [Cyberpublication avant impression].

11. L'Huillier AG, Torriani G, Pigny F, et al. Culture-competent SARS-CoV-2 in nasopharynx of symptomatic neonates, children, and adolescents. Emerg Infect Dis 2020;26:2494-7.

12. Bullard J, Dust K, Funk D, et al. Predicting infectious SARS-CoV-2 from diagnostic samples. Clin Infect Dis 2020;71:2663-6.

13. Wolfel R, Corman VM, Guggemos W, et al. Virological assessment of hospitalized patients with COVID-2019. Nature 2020;581:465-9.

14. DeBiasi RL, Delaney M. Symptomatic and asymptomatic viral shedding in pediatric patients infected with severe acute respiratory syndrome coronavirus 2 (SARS-CoV-2): under the surface. JAMA Pediatr 2021;175:16-8.

15. Fantini MP, Reno C, Biserni GB, et al. COVID-19 and the re-opening of schools: a policy maker's dilemma. Ital J Pediatr 2020;46:79.

16. Heavey L, Casey G, Kelly C, et al. No evidence of secondary transmission of COVID-19 from children attending school in Ireland, 2020. Euro Surveill 2020;25:2000903.

17. Zimmermann $\mathrm{P}$, Curtis $\mathrm{N}$. Coronavirus infections in children including COVID19: an overview of the epidemiology, clinical features, diagnosis, treatment and prevention options in children. Pediatr Infect Dis J 2020;39:355-68.

18. Tönshoff B, Müller B, Elling R, et al. Prevalence of SARS-CoV-2 infection in children and their parents in Southwest Germany. JAMA Pediatr 2021 Jan. 22;e210001. doi: 10.1001/jamapediatrics.2021.0001. [Cyberpublication avant impression].

19. Viner RM, Mytton OT, Bonell C, et al. Susceptibility to SARS-CoV-2 infection among children and adolescents compared with adults: a systematic review and meta-analysis. JAMA Pediatr 2021;175:143-56

20. Lau MSY, Grenfell B, Thomas M, et al. Characterizing superspreading events and age-specific infectiousness of SARS-CoV-2 transmission in Georgia, USA. Proc Natl Acad Sci U S A 2020;117:22430-5. 
21. Lloyd-Smith JO, Schreiber SJ, Kopp PE, et al. Superspreading and the effect of individual variation on disease emergence. Nature 2005;438:355-9.

22. Adam DC, Wu P, Wong JY, et al. Clustering and superspreading potential of SARS-CoV-2 infections in Hong Kong. Nat Med 2020;26:1714-9.

23. Information for Manitobans: COVID-19 dashboard: school-aged cases and school staff cases in Manitoba. Winnipeg: Government of Manitoba. Accessible ici : https://www.gov.mb.ca/covid19/schools/index.html (consulté le 8 févr. 2021).

24. Reports and updates: weekly COVID-19 and seasonal influenza report. Winnipeg: Government of Manitoba. Accessible ici : https://www.gov.mb.ca/health/pub lichealth/surveillance/reports.html (consulté le 8 févr. 2021).

Intérêts concurrents : Lauren Garnett, Kaylie Tran, Alex Bello et James Strong déclarent un financement des services votés du gouvernement du Canada. Aucun autre intérêt concurrent n'a été déclaré.

Cet article a été révisé par des pairs.

Affiliations : Laboratoire provincial Cadham (Bullard, Dust, Hedley, Alexander, Van Caeseele), Santé Manitoba, Département de pédiatrie et de santé de l'enfant (Bullard, Strong, Lee, Van Caeseele, Poliquin), Université du Manitoba; Laboratoire national de microbiologie (Funk, Garnett, Tran, Bello, Strong, Poliquin), Agence de la santé publique du Canada; Département d'anesthésiologie et de médecine (Funk), section des soins intensifs, Université du Manitoba; Département de microbiologie médicale et des maladies infectieuses (Garnett, Tran, Bello, Alexander), Université du Manitoba; Contrôle des maladies transmissibles, Santé publique (Lee), Santé Manitoba; Unité d'épidémiologie et de surveillance (Waruk, Loeppky), Santé Manitoba; Département des sciences en santé communautaire (Loeppky), University of Manitoba, Winnipeg, Man.

Collaborateurs : Tous les auteurs ont contribué à l'élaboration et à la conception du travail ainsi qu'à l'acquisition, l'analyse et l'interprétation des données. Tous les auteurs ont contribué à l'ébauche du manuscrit, ont révisé de façon critique son contenu intellectuel important, ont donné leur approbation finale pour la version destinée à être publiée et endossent l'entière responsabilité de tous les aspects du travail.

Propriété intellectuelle du contenu : Il s'agit d'un article en libre accès distribué conformément aux modalités de la licence Creative Commons Attribution (CC BY-NC-ND 4.0), qui permet l'utilisation, la diffusion et la reproduction de tout médium à la condition que la publication originale soit adéquatement citée, que l'utilisation se fasse à des fins non commerciales (c.-à-d., recherche ou éducation) et qu'aucune modification ni adaptation n'y soit apportée. Voir : https://creativecommons.org/licenses/by-nc-nd/4.0/deed.fr.

Partage des données : Les données comprenant potentiellement des renseignements personnels sur la santé ne peuvent pas être partagées par Santé Manitoba et sont régies par le Comité de la protection des renseignements médicaux selon la Loi sur les renseignements médicaux personnels du Manitoba. Des données anonymisées et dépersonnalisées de façon adéquate pour être rendues publiques pourraient être fournies à des chercheurs sur demande adressée à l'auteur-ressource.

Remerciements : Santé Manitoba, le Laboratoire provincial de Cadham, l'Agence de la santé publique du Canada et le Laboratoire national de microbiologie ont uni leurs efforts pour soutenir ce travail de santé publique en réponse à la pandémie de la COVID-19. Les auteurs veulent aussi remercier les enseignants et les éducateurs qui ont maintenu une certaine normalité pour les enfants du Manitoba ainsi que les enfants eux-mêmes.

Accepté : 30 mars 2021

Correspondance : Jared Bullard, jared.bullard@gov.mb.ca 